\title{
A regional report: Respiratory Syncytial Virus infection in the 2016-17 season
}

\author{
Erin Baroni MD, ${ }^{1}$ Kenzie Daniels MD, ${ }^{2}$ Shawn Reeves RN, MSN, ACPNP, ${ }^{3}$ Mary Frances Flores MLT(ASCP), ${ }^{4}$ \\ Kerrie Pinkney MD, MPH, ${ }^{3}$ Fatma Levent MD $^{3}$
}

\begin{abstract}
Respiratory syncytial virus (RSV) is the most frequent cause of bronchiolitis in children and responsible for more than 125,000 hospitalizations and 250 infant deaths every year. ${ }^{1}$ In this report, an anecdotal increase in RSV bronchiolitis infections in Lubbock, Texas, is discussed.
\end{abstract}

Seasonal outbreaks occur each year, beginning in early winter, peaking in January or February, and ending in spring. Outbreaks depend not only on environmental factors, but also on characteristics specific to the virus. RSV is a non-segmented, single-stranded RNA virus without the ability to reassort genome segments; therefore, it cannot undergo antigenic shifts. However, RSV is dependent on an RNA polymerase, which cannot proofread or edit, so its genome is susceptible to mutations. There are two distinct RSV antigenic subtypes, $A$ and $B$, which differ genealogically. Each of the subtypes has further variability within each of its genomes. RSV A is considered less common, yet responsible for more severe clinical disease and outbreaks due to increased antigenic diversity as compared to RSV B, which is thought to be more common but less frequently associated with outbreaks. $^{2}$

Data were obtained from the clinical virology laboratory of the University Medical Center Hospital in Lubbock, Texas. All patients were identified to be RSV positive by polymerase chain reaction (PCR) detection (Verigene $\left.{ }^{\circledR}\right)$. In the 2016 to 2017 season, experts in Lubbock believe they have seen greater

Corresponding author: Fatma Levent Contact Information: Fatma.levent@ttuhsc.edu DOI: $10.12746 /$ swrccc.v6i22.429
Table. Total number of positive RSV tests for inpatient and outpatient encounters

\begin{tabular}{|l|c|c|}
\hline $\begin{array}{l}\text { Total (inpatient and } \\
\text { outpatient) }\end{array}$ & RSV A+ & RSV B+ \\
\hline December 2016 & 0 & 11 \\
\hline January 2017 & 10 & 26 \\
\hline February 2017 & 8 & 12 \\
\hline Total & 18 & 49 \\
\hline Inpatient only & RSV A+ & RSV B+ \\
\hline December 2016 & 0 & 1 \\
\hline January 2017 & 5 & 8 \\
\hline February 2017 & 5 & 7 \\
\hline Total & $10(56 \%)$ & $16(33 \%)$ \\
\hline
\end{tabular}

numbers and more severe cases of RSV positive bronchiolitis. Although last year's data have not been obtained, this rise is consistent with anecdotal evidence from various hospitals around the country reporting increased numbers of RSV positive bronchiolitis cases requiring inpatient hospitalization. Our data also appear consistent with the traditional understanding of RSV A and RSV B infections. ${ }^{3}$ We had more patients with RSV B, yet a higher percentage of patients with RSV A bronchiolitis were treated as inpatients versus as outpatients (56\% RSV A vs. $33 \%$ RSV B).

More data are needed nationwide to aid in identifying etiologies of increased and more severe RSV bronchiolitis. This information could have implications for management, vaccine studies, reporting standards, and even changes in monoclonal antibody prophylaxis guidelines. ${ }^{4}$ Other factors to consider in our patients include younger age, prematurity, male sex, crowding, lack of breastfeeding, congenital heart 
disease, immunodeficiency, and prophylaxis status, which are all factors that influence susceptibility to RSV infection. ${ }^{5}$ More studies are needed to better identify these characteristics that may have implications for management on a regional, state, and national level.

Keywords: respiratory syncytial virus, bronchiolitis, children, hospitalization

Author affiliation: ${ }^{1}$ Medicine-Pediatrics Residency Program, Brown University, Providence, Rhode Island; ${ }^{2}$ Medicine-Pediatrics Residency Program, Ohio State University, Columbus, Ohio; ${ }^{3}$ Department of Pediatrics, Texas Tech Health Sciences Center, Lubbock, Texas; ${ }^{4}$ Clinical Laboratory, University Medical Center, Lubbock, Texas

Submitted: $12 / 5 / 17$

Accepted: 12/10/17

Conflicts of interest: none

\section{REFERENCES}

1. Piedimonte G, Perez MK. Respiratory syncytial virus infection and bronchiolitis. Pediatrics in Review Dec 2014;35(12): 519-530.

2. SullenderWM. Respiratory syncytial virus genetic and antigenic Diversity. Clinical Microbiology Reviews 2000;13(1):1-15.

3. Rodriguez-Fernandez R, Tapia LI, Yang CF, Torres JP, ChavezBueno S, Garcia C, Jaramillo LM, Moore-Clingenpeel M, Jafri HS, Peeples ME, Piedra PA, Ramilo O, Meijas A. Respiratory syncytial virus genotypes, host immune profiles and disease severity in young children hospitalized with bronchiolitis. J Infect Dis Oct 2017. DOI: 10.1093/infdis/jix543. [Epub ahead of print]

4. American Academy of Pediatrics, Committee on Infectious Diseases and Bronchiolitis Guidelines Committee. Updated guidance for palivizumab prophylaxis among infants and young children at increased risk of hospitalization for respiratory syncytial virus infection. Pediatrics Aug 2014;134(2):415-20.

5. Manzoni P, Figueras-Aloy J, Simones EAF, Checchia PA, Fauroux, Bont L, Paes B, Carbonell-Estrany X. Defining incidence and associated morbidity and mortality of severe respiratory syncytial virus infection among children with chronic diseases. Infect Dis Ther Sep 2017;6(3):383-411. 